\title{
Entre el Mediterráneo y el Atlántico: una travesía histórica y cultural
}

\section{Sergio Arturo Vargas Matías}

Universidad Autónoma de Aguascalientes, Aguascalientes, México, sergiovargasm@gmail.com

\begin{abstract}
This work refers to the analysis of the process of transculturation which meant the transfer - and application-techniques and concepts from the fortification schools European (primarily Italian and French) to the Viceroyalty of new Spain, and specifically, to the shores and paths of the current state of Veracruz, a province of the Republic of Mexico, where today there are still many fortified buildings built between the 16th and 19th, examples of the work of some of the most renowned representatives of art and technique of engineering military service of the Spanish Crown in America.

Composed of forts, batteries, towers, forts, telefres, magazines and other holdouts, the fortified heritage of Veracruz, it constitutes a diverse catalogue of buildings that respond to the influence of some of the most distinctive schools of fortification, from the of reminiscences medieval, as the of the Quiahuiztlan casafuerte, passing through key bastioned system in the various construction stages of the Fort of San Juan de Ulúa to the fortifications built in the route by Xalapa to Veracruz Mexico road under the aegis of the precepts of the Montalembert Marshal.

Currently, these monuments are not only an example of military technology and construction advancements of the time, they also represent a unique testament to the political and social processes that defined the relations between Spain and its possessions in America during the stages of conquest, colonization and independence, so study and historical and cultural importance cannot can be detached from the long process of construction of the national States that throughout the tumultuous 19th-century foundations of the Spanish Empire.
\end{abstract}

Keywords: transculturation, fortification schools, military engineers.

\section{Introducción}

En el territorio que hoy es México, desde antes de la llegada de los españoles, diversos pueblos indígenas, como los mayas, aztecas y totonacas, construyeron múltiples edificios fortificados que cumplían las mismas funciones defensivas que sus contrapartes europeas, parte de los cuales han llegado hasta nuestros días, como los ubicados en Tulum, Xochicalco, La Quemada, y Tepexi El Viejo. Algunos otros, ejemplos notables de la sofisticación alcanzada por la ingeniería vernácula -ya desaparecidos-, son mencionados en las diversas crónicas escritas por los conquistadores llegados allende los mares. Al respecto, Rodríguez Viqueira cita a Cortés, quien en sus Cartas de relación, dice que “...y en un cerro muy alto está la casa del señor con la mejor fortaleza que hay en la mitad de España, y mejor cercada de muro y barbacanas y cavas...".

De acuerdo con Bravo Almazán, en el territorio que hoy es Veracruz, las fortificaciones 
prehispánicas fueron erigidas preferentemente en algunas poblaciones ubicadas alrededor del Pico de Orizaba, formando un rectángulo "entre el Golfo de México, la Sierra Madre Oriental, Quiahuiztlan al norte y Cuauhtochco al sur", área que en opinión de la autora, ofrecía a los habitantes de tales asentamientos condiciones climáticas óptimas, terrenos fértiles, espacio suficiente y vías de desplazamiento adecuadas para su subsistencia.

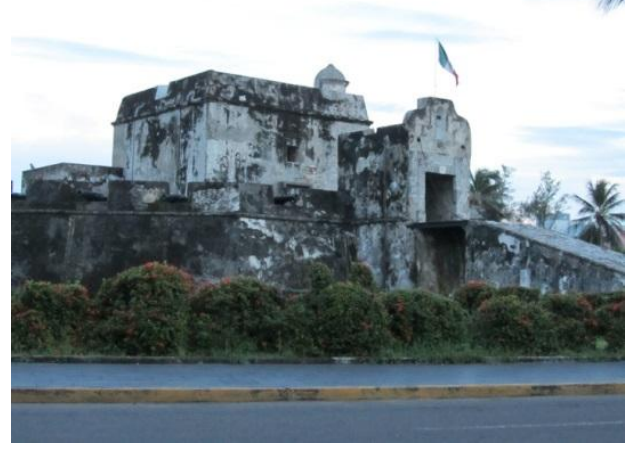

Fig. 1- Baluarte de Santiago (Sergio Vargas, 2011)

Según Bravo Almazán, en Historia antigua de la conquista de México, Manuel Orozco y Berra describe tales asentamientos de la siguiente forma: "Las ciudades fortificadas tenían a veces dos o más recintos fortificados, por otras tantas murallas, completando la defensa interior [de] los teocalli y sus patios cercados...".

Si bien en la actualidad no existen documentos gráficos que permitan conocer de forma detallada la apariencia y disposición espacial de tales construcciones, es posible imaginar su aspecto a partir de los testimonios mencionados, así de los dejados por otros personajes como Antonio Solís, autor de Historia de la conquista de México. Población y progresos de la América septentrional, conocida por el nombre de Nueva España, quien según Ortiz Lanz, al describir una fortificación en Tabasco dice que:

Era el recinto de figura redonda, sin traveses ni otras defensas: y al cerrarse el círculo, dexava hecha la entrada: cruzando, por algún espacio las dos líneas, que componíanvna calle angosta en forma de caracol, donde acomodaban dos o tres garitas, o Castillejos de madera, que estrechavan el passo, y servian de ordinario a sus Centinelas.

A pesar de sus cualidades, las fortificaciones indígenas poco pudieron hacer ante la superioridad tecnológica de los españoles, que encontraron en las armas de fuego y el caballo, junto con la evidente división y el encono que reinaba entre los pueblos originarios, los mejores argumentos para someterlos.

Con la Conquista, el espacio novohispano se fue poblando de fortificaciones que en primera instancia, tenían como propósito defender a los españoles de los ataques de la población nativa, y de igual manera, asegurar la posesión del territorio. A esta lógica responde la primera fortificación española construida en Quiahuiztlan.

A diferencia de las sofisticadas fortalezas que se erigieron posteriormente durante el periodo comprendido entre los siglos XVII y XVIII, la casafuerte de Cortés era un edificio de “...planta cuadrada con cuatro baluartes", cuyo proceso de construcción, según Calderón Quijano, es descrito por Bernal Díaz del Castillo de la siguiente forma:

...hicimos una fortaleza y desde en los cimientos, y en acaballa de tener alta para enmaderar y hechas troneras e cubos $y$ barbacanas dimos tanta priesa, que desde Cortés, que comenzó el primero a sacar tierra a cuestas y piedras e ahondar los cimientos, como todos los capitanes y soldados a la contina entendíamos en ello y trabajábamos por la acabar de presto, los unos en los simientos, y otros en hacer las tapias...

De esta manera, el choque entre el Nuevo y el Viejo mundo, significó no sólo la destrucción del orden politico y social prevaleciente hasta entonces en Mesoamérica, sino la interpenetración y ampliación de los elementos culturales propios de Europa y América, lo que de forma irremediable, se tradujo en el flujo de tales elementos de una civilización a otra.

\subsection{El patrimonio fortificado de Veracruz}

Consumada la derrota del Imperio Mexica y asegurado el dominio ibérico sobre la mayor 
parte del territorio antaño dominado por los aztecas, los españoles se dieron a la tarea de construir múltiples recintos fortificados y edificios logísticos y estratégicos, primero en las costas, y después, tierra adentro, que respondían a una variada tipología de acuerdo con los propósitos de uso y a las condiciones del terreno, así como a la importancia económica y política del espacio que resguardaban.

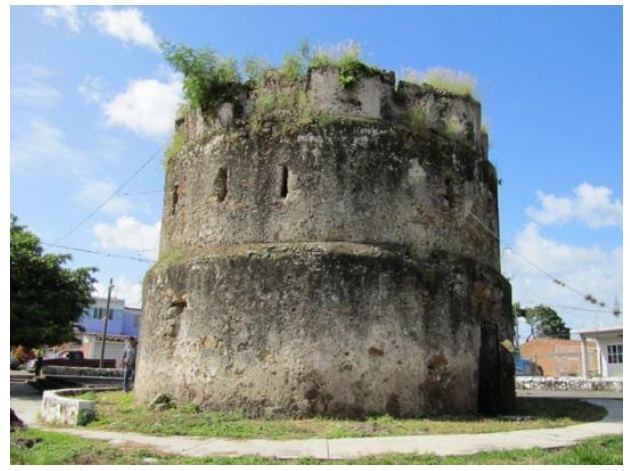

Fig. 2- Fortín de Paso del Macho (Sergio Vargas, 2011)

Lo anterior explica la marcada diferencia entre las fortificaciones de la parte septentrional del virreinato, conocidas como presidios, y las edificadas en las costas adyacentes al Atlántico. Mientras que las primeras fueron erigidas a partir de un diseño constructivo en el que se hallaba ausente cualquier indicio de sofisticación, en la construcción de las segundas se utilizaron de forma prolija los principios del arte y la técnica de la arquitectura militar, a partir de los paradigmas de las escuelas europeas de fortificación, como la italiana, la española y la francesa.

Así, en la edificación de tales obras fue notable la influencia de autores como Antonelli, Vauban y Montalembert, y el trabajo de connotados ingenieros militares como Adrián Boot, Marcos Lucio, Francisco Pozuelo, Jaime Franck, Carlos Blodeaux, Fernando de Pineda, Félix Prosperi, Lorenzo de Solís, Pedro Ponce, Agustín López de la Cámara Alta, Manuel de Santisteban, Miguel Ximénez, Agustín Crame, Ramón Panón, Carlos Duparquet, Segismundo Font y Miguel del Corral, quienes además de participar en la edificación de los fuertes de San Juan de
Úlua y San Carlos de Perote, intervinieron en la construcción de múltiples edificios militares en las costas veracruzanas, así como en la realización de numerosas obras de caminería en las rutas del camino de Veracruz a México, así como en la erección de otras tantas de tipo civil y religioso en diversas partes del virreinato.

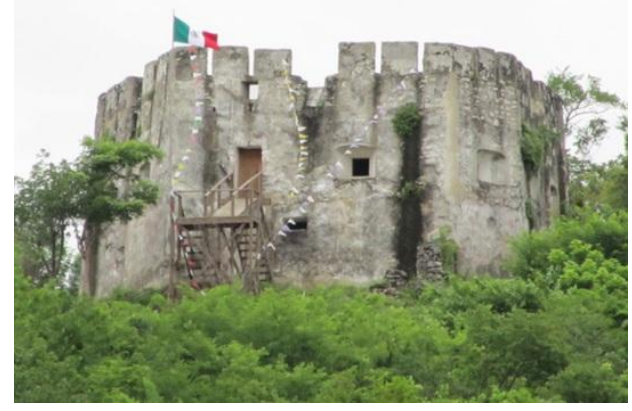

Fig. 3- Fortín de Órdenes Militares de Plan del Río (Sergio Vargas, 2010)

\section{Una clasificación provisional}

Hoy en día, existen numerosas fortificaciones, edificios militares y obras accesorias construidas entre los siglos XVI y XIX que se localizan en algunas partes de las costas veracruzanas y en numerosas poblaciones cercanas a las antiguas rutas -por Orizaba y Xalapa- del camino del puerto de Veracruz a la ciudad de México, que constituyen una parte fundamental del patrimonio cultural material de la nación mexicana.

Con relación a estos inmuebles, es posible proponer una clasificación tentativa, atendiendo a sus particularidades estéticas, funcionales ytécnicas, así como al periodo de su construcción:

- Fortificaciones de reminicencias medievales (siglo XVI):

a) Casafuerte de Cortés (Quiahuiztlan)

- Fortificaciones abaluartadas (siglos XVIIXIX):

a) Fuerte de San Juan de Ulúa (Veracruz)

b) Baluarte de Santiago (Veracruz)

c) Fuerte de San Carlos de Perote

- Fortificaciones de campaña (siglo XIX): 
a) Atalaya (batería) de La Concepción (Puente Nacional)

b) Fortín de la Barranca de Villegas

- Fortificaciones Montalembert (siglos XIX):

a) Fortín de Órdenes Militares (Plan del Río)

b) Fortín de Paso del Macho

- Edificios logísticos, estratégicos y obras accesorias (siglos XVIII y XIX):
a) Polvorines (Fco. I. Madero)
b) Casa de Guardia (Fco. I Madero)
c) Cuartel de lanceros de La Antigua
d) Atarazanas de Veracruz
e) Telefre de Cerro Gordo
f) Telefre de Corral Falso
g) Telefre de Miradores
h) Garitones de Xalapa
i) Atalaya de Tejería

\subsection{Apuntes históricos}

Como es de suponer, la construcción de estos edificios respondió a objetivos y motivaciones de diversa índole, que en síntesis, es posible explicar a partir de los diferentes retos y adversarios a los que estos inmuebles debieron hacer frente, en sintonía con las vicisitudes políticas y militares del periodo en el que fueron erigidos:

- Las fortificaciones levantadas con el fin de proteger a los conquistadores del asedio de la población indígena.

A este periodo corresponde la casafuerte de Quiahuiztlan, que como ya se ha dicho, fue erigida por Cortés en las inmediaciones del asentamiento indígena del mismo nombre en 1519. En la actualidad, de este reducto únicamente sobreviven algunos vestigios, que refieren no sólo la construcción de la fortaleza, sino la de otros edificios levantados por los conquistadores en lo que fue la primera población española en México.

- Las fortificaciones costeras cuyo objetivo primordial fue en primera instancia, proteger los puertos del ataque de los piratas y corsarios y después, de un posible desembarco francés o británico, así como las instalaciones logísticas y estratégicas, construidas para servir como complemento de aquellas.

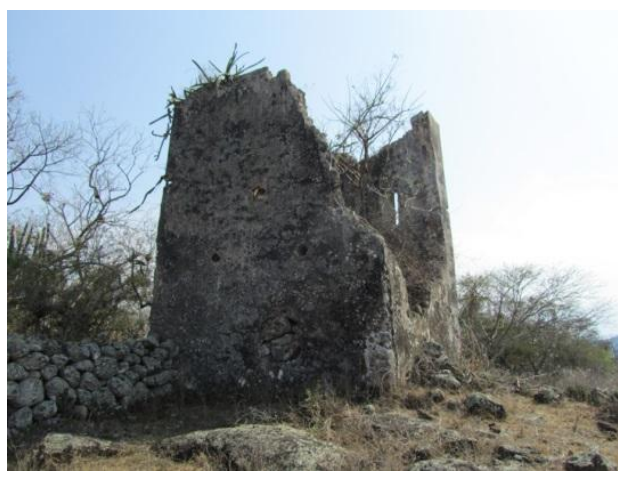

Fig. 4- Telefre de Corral Falso (Sergio Vargas, 2011)

Entre estas, cabe mencionar la que sin duda es la fortaleza más importante de cuantas se edificaron en la Nueva España: el fuerte de San Juan de Ulúa, cuya construcción y mejoras se realizaron prácticamente a lo largo de todo el periodo virreinal; el Baluarte de Santiago, inmueble erigido en el siglo XVII y único sobreviviente del conjunto de baluartes que antiguamente estuvo adosado a la muralla que protegía la plaza de Veracruz; el cuartel de lanceros de La Antigua; y las atarazanas de Veracruz.

- Las fortificaciones y edificios logísticos construidos al interior del territorio que tenían como propósito funcionar como una segunda línea de contención ante la posibilidad de una incursión enemiga hacia la capital del virreinato, una vez superadas las defensas costeras.

A este conjunto, pertenecen el fuerte de San Carlos de Perote, construido entre 1770 y 1776, como parte del proyecto de defensa de la Nueva España instrumentado por el virrey Croix a partir de la visita de Juan de Villalba, al que también responden los polvorines y la casa de guardia construidos durante el mismo periodo en la cercana localidad de Fco. I. Madero.

- Los bastiones e inmuebles estratégicos construidos durante la Guerra de Independencia por insurgentes y realistas en las rutas por Orizaba y Xalapa del Camino Real con el propósito de asegurar el dominio del territorio y las comunicaciones de esta vía. 
Entre estos, cabe mencionar los erigidos por las autoridades novohispanas, como los fortines de Plan del Río y El Órgano, construidos como parte del camino militar establecido por el brigadier Fernando Miyares en 1815; el fortín de la Barranca de Villegas, erigido por el capitán José Ruiz en 1816 para vigilar el paso de convoyes; el fortín de Paso del Macho, edificado en 1818, con el propósito de albergar un destacamento realista para vigilar a un grupo de indultados que se habían establecido con sus familias en las inmediaciones del reducto, lo que con el paso del tiempo, daría origen a la población del mismo nombre; y los telefres de Corral Falso, Miradores, y Cerro Gordo, levantados por Bonifacio de Tosta entre $1818 \mathrm{y}$ 1819.

Respecto de las fortificaciones insurgentes, cabe mencionar la batería de La Concepción, construida en los márgenes del Puente del Rey y arrebatada a los rebeldes por Miyares en 1815.

- Las fortificaciones y obras accesorias construidas por los sucesivos gobiernos mexicanos una vez alcanzada la independencia, con el fin de vigilar los caminos y ciudades y robustecer la capacidad de defensa de la nación, las cuales continuaron siendo levantadas bajo los preceptos de la ingeniería militar europea.

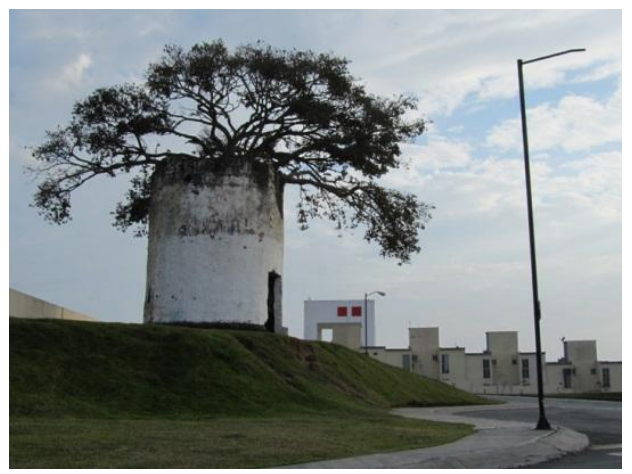

Fig. 5- Atalaya de Tejería (Sergio Vargas, 2012)

En este apartado hay que incluir obras como el fortincillo y la garita de Soledad de Doblado, algunos de los garitones existentes hoy día en Xalapa, y probablemente, la atalaya de Tejería, el bastión de Fortín de las Flores, y el reducto de Antón Lizardo, del cual se ha mencionado fue edificado durante el periodo colonial, sin que hasta el momento se haya localizado constancia documental que acredite su construcción en dicho lapso.

\section{Conclusiones}

A través de estas breves líneas, se ha intentado presentar un bosquejo del proceso de traslación del arte y la técnica de la fortificación europea al continente americano, tomando como vehículos para esta travesía, los edificios que, construidos durante el periodo virreinal y los primeros años del México independiente, sobreviven en diversas localidades de las costas y los caminos de la entidad veracruzana.

$\mathrm{Si}$ bien es cierto que buena parte de estos inmuebles ha sido referida en numerosos trabajos por algunos académicos, cronistas e investigadores independientes, así como en diversas publicaciones editadas por las instituciones dedicadas al fomento $y$ la investigación de las artes y la cultura, hasta el momento, no existe un inventario que compendie en su totalidad el conjunto de estos monumentos, varios de los cuales en la actualidad se encuentran en riesgo de desaparecer.

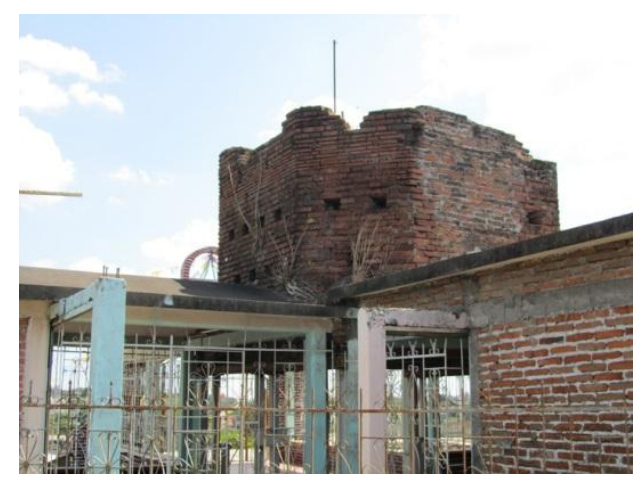

Fig. 6- Forticincillo de Soledad de Doblado (Sergio Vargas, 2011)

Esta situación puede explicarse como una consecuencia que se deriva de distintos factores, entre los que destacan el desconocimiento que prevalece respecto de estos edificios incluso en los círculos académicos, y el escaso interés que muestran los organismos públicos encargados de 
su protección, así la apatía e indiferencia de la sociedad en general, lo que se traduce en la carencia de políticas y acciones efectivas para su conservación y puesta en valor.

Por tanto, más allá de los aspectos académicos, el objetivo fundamental de la presente comunicación es contribuir, así sea de forma mínima, al conocimiento y difusión del patrimonio fortificado de Veracruz, como primer paso para su protección, conservación y puesta en valor.

\section{Notas}

Desde estas líneas, queremos expresar nuestro reconocimiento al Consejo Nacional de Ciencia y Tecnología por el apoyo brindado para llevar a cabo la investigación en la que se sustenta el presente texto, así como nuestro agradecimiento a la Universidad Autónoma de Aguascalientes por su contribución para la presentación de esta comunicación.

\section{Referencias}

Abad, Tomás y Pilar Chías (2011). El patrimonio fortificado. Cádiz y el Caribe: una relación trasatlántica. Universidad de Alcalá. Madrid.

Archer, Christon. (1983). El ejército en el México borbónico, 1760-1810. Fondo de Cultura Económica. México.

Bravo, Verónica (2011). "Una aproximación al estudio de las fortificaciones prehispánicas en el centro de Veracruz" en Estudios Mesoamericanos. Instituto de Investigaciones Filológicas-UNAM. México.

Broca, Abraham y otros. (2007). Itinerario cultural. Camino Real de Veracruz a Perote. Gobierno del Estado de Veracruz/Universidad Veracruzana/Instituto Veracruzano de Cultura/Ayuntamiento de Perote/Ayuntamiento de Veracruz. Veracruz.

Calderón, José. (1984). Historia de las fortificaciones en Nueva España. Consejo Superior de Investigaciones Científicas/Gobierno del Estado de Veracruz. Madrid.

Chaunu, Pierre. (1960). "Veracruz en la segunda mitad del siglo XVI y primera del siglo XVII" en Historia Mexicana. El Colegio de México. México.

Dembicz, Katarzyna. (2015). “Multidimensionalidad de América Latina ¿Efecto mundialización?” en $I V$ Encuentro Internacional AHILA 2015. Instituto Veracruzano de la Cultura. Veracruz.

Fernández, Ángel y otros. (2010). Fortalezas históricas de Veracruz. Secretaría de Educación del Gobierno del Estado de Veracruz. México.

García, Alfonso. (2011). "Fortín de órdenes militares de Plan del Río, Veracruz" en Investigaciones culturales. Universidad Veracruzana/Instituto de Antropología de la Universidad Veracruzana/Universidad de Xalapa. Xalapa.

González, Jorge y otros. (2009). Corpus urbanístico. Arquitectura militar. Fortificaciones costeras de México en los archivos españoles. Universidad Autónoma Metropolitana/Instituto Nacional de Antropología e Historia/Consejo Nacional para la Cultura y las Artes/Embajada de España en México. México.

González, Martín. (2007). "El rey Revillagigedo y la defensa del puerto de Veracruz, 1789-1794” en Relaciones. El Colegio de Michoacán. Zamora.

Ortiz, José. (1993). Arquitectura militar de México. Secretaria de la Defensa Nacional. México.

Ortiz, Juan. (2010). El teatro de la guerra. Veracruz 1750-1825. Universidad Veracruzana/Universitat Jaume I. Xalapa.

Trens, Manuel. (1955). Historia de la H. ciudad de Veracruz y de su ayuntamiento. Archivo General de la Nación. México.

Vargas, Sergio. (2011). "El Camino Real de Veracruz: pasado, presente y futuro". Ponencia presentada en el XVII Congreso Internacional de la International Association for Intercultural Communication Studies (IAICS). San Cristóbal de las Casas. 
(2012). "La ruta de las fortificaciones históricas de Veracruz como opción de desarrollo comunitario a través del turismo alternativo". Ponencia presentada en el XXXV Encuentro Internacional RNIU 2012. San Luis Potosí.

(2013). "El sistema fortificado del Camino Real de Veracruz: legado de la técnica y el arte europeos en la Nueva España". Ponencia presentada en el IX Encuentro Internacional de Historiografía. Transculturación: espacios y tiempos. México.

(2013). "Guardianes a pie firme: el sistema fortificado de defensa del camino de Veracruz a México". Ponencia presentada en el VII Seminario de Historia Regional. Historiografía Regional e Historia Cultural. Nuevas Aportaciones. Aguascalientes.

(2013). "Guardianes a pie firme: el sistema fortificado de defensa del camino de Veracruz a México". Ponencia presentada en el Coloquio Internacional: El Patrimonio desde las Ciencias Sociales y las Humanidades. San Luis Potosí.

(2013). "Escenarios de conflicto, custodios de la historia: el patrimonio fortificado del camino de Veracruz a México. Apuntes para su estudio". Ponencia presentada en el III Foro de Investigación sobre Arquitectura y Urbanismo Militar en Iberoamérica. México.

Vázquez, José. Telegrafía óptica en México y los telefres. Consultado el 15 de septiembre de 2013. Disponible en: htttp://www.telefre.com/Documentos/historia_telefre.pdf 
